\title{
Prevalence, Risk Factors, and Etiology of Extubation Failure in Pediatric Patients After Cardiac Surgery
}

\author{
Asaad G. Beshish10 Michael P. Fundora1,* \\ Fawwaz R. Shaw ${ }^{5}$ Kevin O. Maher ${ }^{1}$ Michael Wolf $^{1}$
}

Address for correspondence Asaad Beshish, MD, Division of Cardiology, Department of Pediatrics, Emory University School of Medicine, Children's Healthcare of Atlanta, Sibley Heart Center Cardiology, 2835 Brandywine Road, Suite 400, Atlanta, GA 30341, United States

(e-mail: beshisha@kidsheart.com; abeshis@emory.edu).

\section{Keywords \\ - extubation failure \\ - congenital heart disease \\ - cardiac surgery}

In this article, our primary objective was to investigate the prevalence and etiology of extubation failure (EF) in patients following cardiac surgery for congenital heart disease. Secondarily, we examined the association of different risk factors with EF. This was single-center retrospective study in a 27-bed cardiac intensive care unit at a quaternary children's hospital. All patients between 0 and 18 years of age who underwent congenital cardiac surgery from January 2008 to September 2019 were included. During the study period, among 8,750 surgical encounters, 257 (2.9\%) failed extubation, defined as reintubation within 48 hours from extubation. EF patients were younger, smaller, more likely to have genetic syndromes, higher Society of Thoracic Surgeons and the European Association for Cardio-Thoracic Surgery (STAT) mortality scores, single-ventricle physiology, longer cardiopulmonary bypass (CPB) and crossclamp (XC) times, longer mechanical ventilation (MV) duration, and higher mortality ( $p$ $<0.05)$. In a univariate analysis, EF patients when compared to matched controls by age, gender, and STAT score, and genetic syndrome, particularly heterotaxy, were associated with increased odds of EF $(p<0.05)$. In a multivariable logistic regression of the entire cohort, the presence of any genetic syndrome was associated with higher odds of EF ( $p<0.05)$. In a subgroup of neonates, a univariate analysis was performed and multivariable analysis was attempted, but both did not achieve statistical significance. In summary, EF after congenital cardiac surgery is associated with younger age, lower weight, single-ventricle physiology, longer CPB and XC times, longer duration of MV, and genetic syndromes. Patients failing extubation have increased morbidity and mortality. Recognition of these risk factors may provide clinicians the ability to identify patients at high risk allowing for timely intervention to limit adverse outcomes.

Co-first author

received

October 14, 2021

accepted after revision

December 12, 2021
DOI https://doi.org/

$10.1055 / \mathrm{s}-0041-1742253$. ISSN 2146-4618.

\footnotetext{
(C) 2022. The Author(s).

This is an open access article published by Thieme under the terms of the Creative Commons Attribution-NonDerivative-NonCommercial-License, permitting copying and reproduction so long as the original work is given appropriate credit. Contents may not be used for commercial purposes, or adapted, remixed, transformed or built upon. (https://creativecommons.org/ licenses/by-nc-nd/4.0/) Georg Thieme Verlag KG, Rüdigerstraße 14, 70469 Stuttgart, Germany
} 


\section{Introduction}

The postoperative management of children undergoing congenital heart surgery has evolved with shorter durations of mechanical ventilation (MV) and lengths of stay (LOS). ${ }^{1-10}$ Early extubation, defined as extubation either in the operating room or within 6 hours of arrival to the cardiac intensive care unit (CICU), is an approach used to limit the duration of MV and contribute to improved outcomes. ${ }^{1-4}$ There remains a subset of patients that fail extubation, thus causing a setback in their recovery. ${ }^{11-13}$ Extubation failure (EF) in children is quite common and is seen in 22 to $28 \%$ of premature babies, 15 to $20 \%$ of critically ill children, and 3 to $10 \%$ of children after cardiac surgery. ${ }^{5,6,8,11,14-17}$ Extubation in neonates poses the greatest challenge after cardiac surgery due to immature respiratory function, small airways, neurological factors including sedation, and nutritional status to name a few. ${ }^{3,5,12,15,16,18}$ In both adults and children, unsuccessful extubation requiring emergent reintubation may cause significant hemodynamic instability, airway trau$\mathrm{ma}$, prolonged duration of intensive care unit stay, and overall increasing the risk of morbidity and mortality. ${ }^{5,16-21}$

This study aimed to investigate the prevalence and etiology of EF in the patient following cardiac surgery for congenital heart disease (CHD) as our primary outcome. Secondarily, we sought to examine the association of different risk factors with $\mathrm{EF}$.

\section{Materials and Methods}

\section{Cohort Selection}

This single-center retrospective cohort study included all pediatric patients, 0 to 18 years old, who underwent cardiac surgery for repair or palliation of CHD from January 1, 2008 to September 30, 2019, at Children's Healthcare of Atlanta, a free-standing university-affiliated quaternary children's hospital. An internal surgical database was queried, and eligible surgical encounters were identified. Preterm neonates with the corrected gestational age of less than 35 weeks, patients undergoing patent ductus arteriosus ligation, epicardial pacemaker implantation, pericardial window as their sole procedure, and patients with the diagnoses of cardiomyopathy, cardiac tumors, and myocarditis placed on extracorporeal life support were all excluded. This project was reviewed and approved by the Children's Healthcare of Atlanta Institutional Review Board (IRB\# 00000339). Informed consent was waived.

Demographics (age, weight, gender, and race), clinical characteristics (CICU-LOS, postoperative-LOS, hospital-LOS, duration of MV, genetic syndrome, and chromosomal abnormalities), and operative variables (cardiopulmonary bypass [CPB] time and cross-clamp [XC] time) were collected. EF was defined as reintubation within 48 hours from extubation. Once patients who failed extubation were identified $(n=257)$, an attempt was made to match controls in the ratio of 2:1 based on age, gender, and Society of Thoracic Surgeons and the European Association for Cardio-Thoracic Surgery (STAT) mortality scores category. Total number of patients that were able to match were in the ratio of 231 (cases):462 (controls). Surgical procedures were categorized by complexity based on the STAT mortality scores. ${ }^{22}$ Duration of MV was defined as the duration of time from intubation to the first extubation attempt. Mortality at discharge was defined as (1) all deaths occurring during the hospitalization in which the operation was performed, even if after 30 days, and (2) all deaths occurring after discharge from the hospital, but before the end of the 30th postoperative day. ${ }^{22}$

\section{Airway Management}

All patients were endotracheally intubated with cuffed endotracheal tubes and placed on MV on admission from the operating room. Depending on the peri-operative and patient factors, patients were placed on fast-track extubation protocol. The protocol was initiated once patients were spontaneously breathing and extubation readiness was determined. Patients were then placed on spontaneous breathing trials (SBT) using continuous positive airway pressure with pressure support (CPAP/PS). Following a successful SBT, patients were extubated to non-invasive respiratory support at the discretion of the $\mathrm{CICU}$ physician ranging from nasal cannula (NC), humidified high-flow nasal cannula (HFNC), to noninvasive positive pressure ventilation (NIPPV) with either CPAP or bilevel ventilation (BiPAP). Respiratory support following extubation was dichotomized to NC/HFNC or NIPPV. NIPPV included both CPAP and BiPAP. Patients failing extubation were reintubated when meeting at least 1 of the following criteria: (1) clinical signs of respiratory fatigue and/or severe respiratory distress; (2) worsening hypercarbia or hypoxemia; (3) inability to clear airway/oral secretions; (4) hemodynamic decompensation; (5) cardiorespiratory arrest; and (6) inability to maintain adequate airway patency due to neurologic impairment. These criteria are similar to what has been used in a prior report by Miura et al. ${ }^{5}$

\section{Statistical Analysis}

Statistical analysis was performed using SAS version 9.4 (Cary, NC, United States), and statistical significance was assessed at the 0.05 level. The normality of continuous variables was assessed using histograms, normal probability plots, and the Anderson-Darling test for normality. Descriptive statistics were calculated for all variables of interest and included medians and interquartile ranges (IQR) and counts and percentages, when appropriate. Characteristics of subjects who failed extubation (EF-group) and those who did not fail extubation (non-EF group) were compared using chisquare tests for categorical variables or Fisher's exact test when expected cell counts were small $(<5)$ and a Wilcoxon rank-sum test for continuous variables.

To minimize confounding variables, we matched EF to non-EF patients (1:2) by age category, gender, and STAT category and yielded 231 cases and 462 controls. Univariate logistic regression and multivariable logistic regression were used to obtain estimates of the association between EF and each candidate predictor. Predictors that were significant at the univariate level $(p<0.20)$ and had sufficient sample size 
were included in multivariable modeling. Both unadjusted and adjusted odds ratios (aORs) and 95\% confidence intervals (CIs) were constructed for each logistic regression model. The diagnostic performance for the prediction of extubation was assessed using the area under the receiver operating characteristic curve. Multivariable logistic regression of the neonate-only cohort was attempted but did not achieve statistical significance.

\section{Results}

\section{Patient Characteristics}

There were 8,750 eligible cardiac surgical encounters from 6,247 unique patients during the study period. Patient characteristics are presented in -Table 1. The median age was 6.5 months (IQR 1.5-51.7), and the median weight was $6.5 \mathrm{~kg}$ (IQR 3.8-15.7). Neonates and infants comprised 5,240 encounters (59.9\%). Operations with STAT scores of 4 or 5 comprised 2,116 (27.6\%) of all surgical encounters. The median duration of CPB and XC time were 94 minutes (IQR 65-137) and 47 minutes (IQR 21-75), respectively. The median CICU-LOS was 4.2 days (IQR 2.1-7.7), and the median duration of MV was 22.15 hours (IQR 11.6-74.0). Chromosomal abnormalities were present in $18.7 \%$ and diagnosed genetic syndromes in $22.4 \%$. The most common syndromes were Down syndrome ( $n=638,7.3 \%$ ), DiGeorge syndrome $(n=295,3.4 \%)$, and heterotaxy syndrome $(n=334,3.8 \%)$ (-Table 1).

\section{Extubation Failure}

EF was defined as reintubation within 48 hours from the time of extubation. EF occurred in 257 (2.9\%) encounters. Neonates had an EF rate of 5.9\%, while infants had a 3.0\% EF rate. By far the majority of patients in the EF group were extubated to NC or HFNC $(90.3 \%, n=232)$. Patients in the EF group were younger (median age 1.7 vs. 6.7 months, $p<0.001$ ) and smaller (median weight 4 vs. $6.6 \mathrm{~kg}, p<0.001$ ) than the non-EF group. EF patients had longer CPB (median 126.5 vs. 93 minutes, $p<0.001$ ) and XC times (median 59 vs. 47 minutes, $p$ 0.002). EF patients had a longer CICU- and hospital-LOS and longer duration of MV prior to initial extubation (-Table 1). EF patients were more likely to have a higher STAT score of 4 and 5 (41.9\% vs. 22.4, and $15.0 \%$ vs. $4.3 \%, p<0.001)$, as well genetic syndromes $(33.0 \%$ vs. $21.1 \%, p 0.003)$. EF patients had a higher percentage of mortality ( $8.9 \%$ vs. $5.3 \%, p<0.001$ ) when compared with the non-EF patients (-Table $\mathbf{1}$ ).

This most common etiology of EF in our cohort was atelectasis in $32.3 \%(n=83)$, followed by poor gas exchange in $16.7 \%(n=43)$, low cardiac output and hemodynamic instability in $16.3 \%(n=42)$, upper airway obstruction in $14.8 \%(n=38)$, and pulmonary congestion in $10.9 \%$ $(n=28)$ (-Fig. 1). The most common cardiac lesion category in the EF group was single-ventricle physiology $(n=69)$, followed by tetralogy of fallot $(n=30)$, interrupted aortic $\operatorname{arch} \pm \operatorname{VSD}(n=27)$, atrioventricular septal defect $(n=20)$, D-transposition of the great arteries $(n=16)$, and double outlet right ventricle $(n=15)$ (-Table 2). A detailed list outlining the etiology of EF and the different cardiac lesion categories are shown in -Fig. 1 and - Tables 2.

\section{Predictors for EF}

Univariate analysis comparing EF patients $(n=231)$ to matched controls $(n=462)$ in the ratio of $2: 1$ by age, gender, and STAT category showed that risk factors such as CPB, XC time, single-ventricle physiology, duration of MV, and mode of respiratory support post-extubation were not associated with increased odds of EF (-Table 3). Our analysis did demonstrate that CICU-LOS (OR 1.02, 95\% CI 1.01-1.03, $p$ $<0.001$ ), postoperative-LOS (OR 1.01, 95\% CI 1.01-1.02, $p$ $<0.001$ ), hospital-LOS (OR 1.01, 95\% CI 1.01-1.02, $p<0.001$ ), and any genetic syndromes (OR 1.61, 95\% CI 1.13-2.30, $p=0.009$ ), in particular heterotaxy syndrome (OR 2.68, $95 \%$ CI 1.29-5.58, $p=0.008$ ), were all associated with increased odds of EF ( - Table 3). Multivariable logistic regression was performed and demonstrated that CICU-LOS (OR $1.02,95 \% \mathrm{CI} 1.01-1.03, p<0.001)$ and the presence of a genetic syndrome (OR 1.50, 95\% CI 1.04-2.16, $p$ 0.031) were associated with increased odds of EF ( - Table 3 ).

\section{Neonatal Subgroup Analysis}

Univariate analysis focusing on neonates (age $<29$ days) was performed comparing neonates who failed extubation $(n=106)$ matched to controls $(n=212)$ in the ratio of $2: 1$ by age, gender, and STAT category which demonstrated that CICU-LOS (OR 1.02, 95\% CI 1.01-1.03, $p<0.001$ ), postoperative-LOS (OR 1.01, 95\% CI 1.01-1.02, $p<0.001$ ), hospital-LOS (OR 1.01, 95\% CI 1.01-1.02, $p<0.001$ ) were associated with increased odds of EF (-Table 4). Multivariable logistic regression analysis of the neonate-only cohort was attempted but did not achieve statistical significance.

\section{Discussion}

This is the largest single-center study examining EF after congenital cardiac surgery. EF is relatively uncommon after surgery for CHD. Our EF rate of $2.9 \%$ is slightly lower than prior reports ranging from 3 to $10 \%{ }^{4-7,10,17}$ This rate reflects our practice of balancing aggressive and conservative extubation strategies as well as the implementation of SBT to support extubation readiness. Of the patients failing extubation, neonates and infants represented 83.3\% (214/257). Neonates had the highest rates of EF $(5.9 \%)$ in our cohort. Although the duration of mechanical ventilation in our study is comparable to other reports, our neonatal EF rate is strikingly lower than prior reports of neonatal EF ranging from 10 to $27 \%{ }^{5,11,12,14,15,17,23}$ Contributing factors resulting in neonatal EF include but are not limited to anatomic and physiologic characteristics such as smaller airways that may be vulnerable to mucosal edema. Other factors that are not specific to neonates such as acid-base disturbances and hemodynamic instability due to a prolonged peri-operative course or residual lesions can play important roles in EF. ${ }^{15}$ Additionally, neonates undergoing cardiac surgery are likely to present with more complex lesions, post-anesthesia apnea, functional immaturity of different organ systems, and 
Pediatric Patients After Cardiac Surgery Beshish et al.

Table 1 Patient characteristics of the entire cohort and stratified into extubation failure group and non-extubation failure group

\begin{tabular}{|c|c|c|c|c|}
\hline \multirow[t]{2}{*}{ Variables } & Overall cohort & Non-EF group & EF group & \multirow[t]{2}{*}{$p$-Value } \\
\hline & $n=8,750(\%)$ & $n=8,493(\%)$ & $n=257(\%)$ & \\
\hline \multicolumn{5}{|l|}{ Age group } \\
\hline Neonates & $1,957(22.4 \%)$ & $1,842(21.7 \%)$ & $115(44.8 \%)$ & \multirow[t]{5}{*}{$<0.001$} \\
\hline Infants & $3,283(37.5 \%)$ & $3,184(37.5 \%)$ & 99 (38.5\%) & \\
\hline 1 to $3 y$ & $885(10.1 \%)$ & $866(10.2 \%)$ & $19(7.4 \%)$ & \\
\hline 3 to $5 y$ & $646(7.4 \%)$ & 640 (7.5\%) & $6(2.3 \%)$ & \\
\hline 5 to $18 \mathrm{y}$ & $1,979(22.6 \%)$ & $1,961(23.1 \%)$ & $18(7.0 \%)$ & \\
\hline Age (months) & $6.5(1.5-51.7)$ & $6.7(1.7-53.8)$ & $1.7(0.2-5.5)$ & $<0.001$ \\
\hline Weight (kg) & $6.5(3.8-15.7)$ & $6.6(3.9-16.0)$ & $4(3.1-6.4)$ & $<0.001$ \\
\hline \multicolumn{5}{|l|}{ Race } \\
\hline Asian & $295(3.4 \%)$ & $288(3.4 \%)$ & $7(2.7 \%)$ & \multirow[t]{5}{*}{0.345} \\
\hline Black/African American & $2,916(33.3 \%)$ & $2,815(33.1 \%)$ & $101(39.3 \%)$ & \\
\hline Caucasian & $5,017(57.3 \%)$ & $4,882(57.5 \%)$ & $135(52.5 \%)$ & \\
\hline Hispanic & $173(2.0 \%)$ & $169(2.0 \%)$ & $4(1.6 \%)$ & \\
\hline Other & $349(4.0 \%)$ & $339(4.0 \%)$ & $10(3.9 \%)$ & \\
\hline \multicolumn{5}{|l|}{ Gender } \\
\hline Female & $3,959(45.3 \%)$ & $3,845(45.3 \%)$ & $114(44.4 \%)$ & \multirow[t]{2}{*}{0.772} \\
\hline Male & $4,791(54.8 \%)$ & $4,648(54.7 \%)$ & $143(55.6 \%)$ & \\
\hline \multicolumn{5}{|l|}{ STAT score $(n=7,661)$} \\
\hline 1 & $2,406(31.4 \%)$ & $2,378(32.0 \%)$ & $28(12.0 \%)$ & \multirow[t]{5}{*}{$<0.001$} \\
\hline 2 & $2,215(28.9 \%)$ & $2,171(29.2 \%)$ & $44(18.8 \%)$ & \\
\hline 3 & $924(12.1 \%)$ & $895(12.1 \%)$ & $29(12.4 \%)$ & \\
\hline 4 & $1,761(23.0 \%)$ & $1,663(22.4 \%)$ & $98(41.9 \%)$ & \\
\hline 5 & $355(4.6 \%)$ & $320(4.3 \%)$ & $35(15.0 \%)$ & \\
\hline \multicolumn{5}{|l|}{ Single ventricle } \\
\hline No & $6,924(79.1 \%)$ & $6,753(79.5 \%)$ & $171(66.5 \%)$ & \multirow[t]{2}{*}{$<0.001$} \\
\hline Yes & $1,826(20.9 \%)$ & $1,740(20.5 \%)$ & $86(33.5 \%)$ & \\
\hline CPB time $(\min )^{\mathrm{a}}$ & $94(65-137)$ & $93(64-135.5)$ & $126.5(93-174)$ & $<0.001$ \\
\hline $\mathrm{XC}$ time $(\min )^{\mathrm{b}}$ & $47(21-75)$ & $47(21-74)$ & $59(30-86)$ & 0.002 \\
\hline CICU LOS (d) & $4.2(2.1-7.7)$ & $4.2(2.1-7.7)$ & $15.4(8.4-30.8)$ & $<0.001$ \\
\hline Hospital LOS (d) & $7(4-18)$ & $7(4-17)$ & $28(14-61)$ & $<0.001$ \\
\hline \multicolumn{5}{|l|}{ Chromosomal abnormality } \\
\hline No & $7,114(81.3 \%)$ & $6,905(81.3 \%)$ & $209(81.3 \%)$ & \multirow[t]{2}{*}{0.993} \\
\hline Yes & $1,636(18.7 \%)$ & $1,588(18.7 \%)$ & $48(18.7 \%)$ & \\
\hline \multicolumn{5}{|l|}{ Genetic syndrome } \\
\hline No & $6,793(77.6 \%)$ & $6,613(77.9 \%)$ & $180(70.0 \%)$ & \multirow[t]{2}{*}{0.003} \\
\hline Yes & $1,957(22.4 \%)$ & $1,880(22.1 \%)$ & $77(30.0 \%)$ & \\
\hline \multicolumn{5}{|l|}{ Type of genetic syndrome } \\
\hline Down syndrome & $638(7.3 \%)$ & $626(7.4 \%)$ & $12(4.7 \%)$ & \multirow[t]{5}{*}{$<0.001$} \\
\hline DiGeorge syndrome & $295(3.4 \%)$ & $279(3.3 \%)$ & $16(6.2 \%)$ & \\
\hline Heterotaxy syndrome & $334(3.8 \%)$ & $316(3.7 \%)$ & $18(7.0 \%)$ & \\
\hline Others & $1,109(12.7 \%)$ & $1,070(12.6 \%)$ & $39(15.2 \%)$ & \\
\hline None & 6,374 (72.9\%) & $6,202(73.0 \%)$ & 172 (66.9\%) & \\
\hline
\end{tabular}


Pediatric Patients After Cardiac Surgery Beshish et al.

Table 1 (Continued)

\begin{tabular}{|c|c|c|c|c|}
\hline \multirow[t]{2}{*}{ Variables } & Overall cohort & Non-EF group & EF group & \multirow[t]{2}{*}{$p$-Value } \\
\hline & $n=8,750(\%)$ & $n=8,493(\%)$ & $n=257(\%)$ & \\
\hline Duration of MV $(h)^{c}$ & $22.2(11.6-74.0)$ & $21.8(11.3-71.7)$ & $96.3(25.9-221.3)$ & $<0.001$ \\
\hline \multicolumn{5}{|c|}{ Respiratory support post-extubation } \\
\hline $\mathrm{NC} / \mathrm{HFNC}$ & $232(90.3 \%)$ & - & $232(90.3 \%)$ & \\
\hline NIPPV & $25(9.7 \%)$ & - & $25(9.7 \%)$ & \\
\hline \multicolumn{5}{|l|}{ Mortality at discharge } \\
\hline Alive & $8,276(94.6 \%)$ & $8,042(94.7 \%)$ & $234(91.1 \%)$ & \multirow[t]{2}{*}{$<0.001$} \\
\hline Dead & $474(5.4 \%)$ & $451(5.3 \%)$ & $23(8.9 \%)$ & \\
\hline
\end{tabular}

Abbreviations: CICU-LOS, cardiac intensive care unit length of stay; CPB, cardiopulmonary bypass; EF, extubation failure; HFNC, high-flow nasal cannula; Hospital-LOS, hospital length of stay; MV; mechanical ventilation; NC, nasal cannula; NIPPV, non-invasive positive pressure ventilation; STAT scores, Society of Thoracic Surgeons and the European Association for Cardio-Thoracic Surgery mortality scores; XC, cross-clamp.

Results depicted in $n$ (percent) and median (interquartile range).

Note: Bolded p-Values are statistically significant.

${ }^{a}$ Note that CPB time was estimated in a smaller cohort of $n=6,260$ surgical encounters.

${ }^{b}$ Note that cross-clamp time was estimated in a smaller cohort of $n=6,708$ surgical encounters.

'Duration of mechanical ventilation is the time from preoperative intubation to the first attempt of extubation following congenital cardiac surgery.

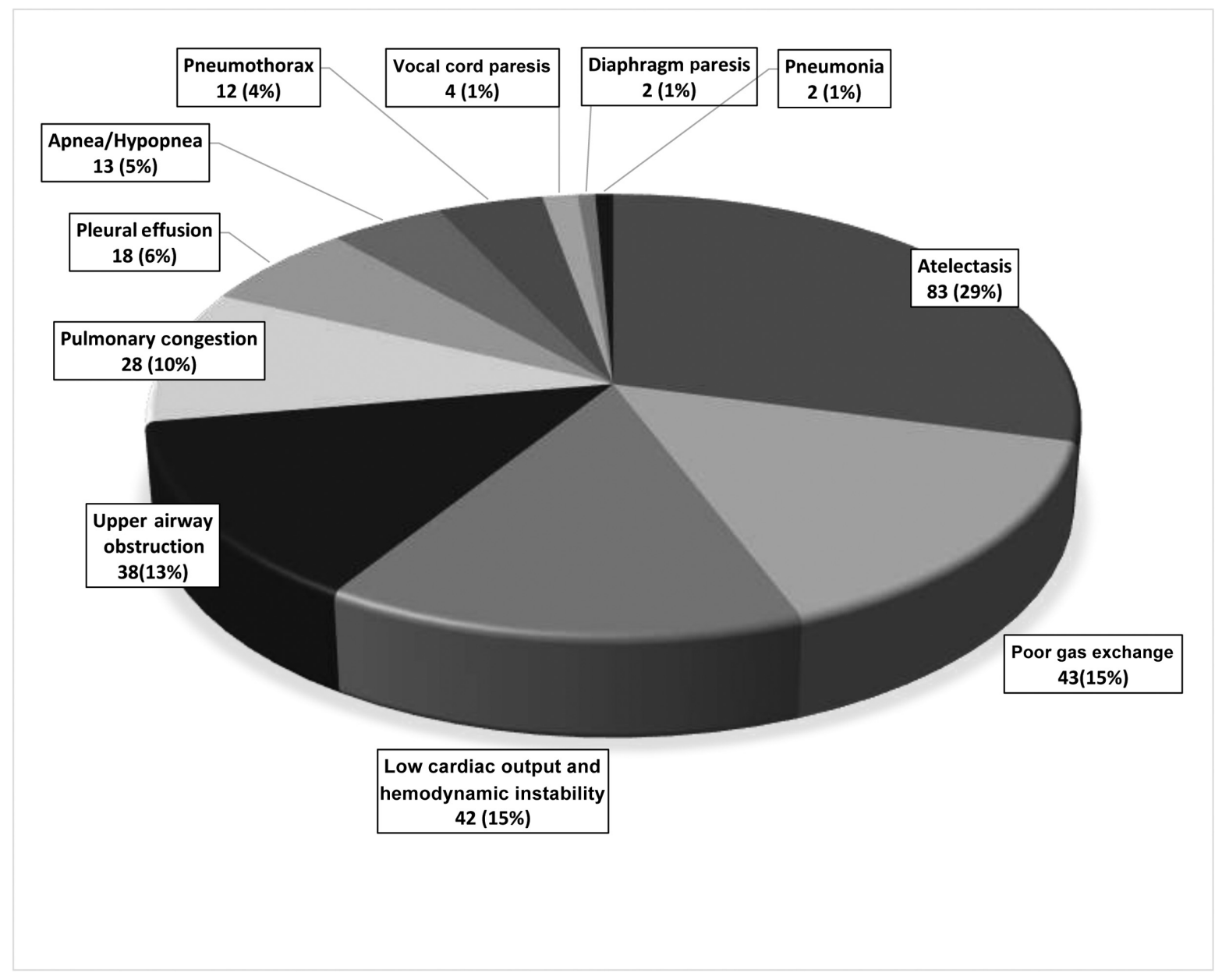

Fig. 1 Etiology of extubation failure. 
Pediatric Patients After Cardiac Surgery Beshish et al.

Table 2 Extubation failures by broad cardiac lesions categories

\begin{tabular}{|l|l|}
\hline Category & Count $(\boldsymbol{n}=\mathbf{2 5 7})$ \\
\hline Single ventricle physiology & $69(26.8 \%)$ \\
\hline Tetralogy of fallot & $30(11.7 \%)$ \\
\hline Interrupted aortic arch \pm VSD & $27(10.5 \%)$ \\
\hline Atrioventricular septal defects & $20(7.8 \%)$ \\
\hline $\begin{array}{l}\text { D-transposition of the great } \\
\text { arteries } \pm \text { VSD }\end{array}$ & $16(6.2 \%)$ \\
\hline Double outlet right ventricle & $15(5.8 \%)$ \\
\hline Ventricular septal defects (VSD) & $9(3.5 \%)$ \\
\hline $\begin{array}{l}\text { Coarctation of the aorta and arch } \\
\text { hypoplasia } \pm \text { VSD }\end{array}$ & $9(3.5 \%)$ \\
\hline $\begin{array}{l}\text { Pulmonary atresia, intact ventricu- } \\
\text { lar septum (PA/IVS) }\end{array}$ & $9(3.5 \%)$ \\
\hline $\begin{array}{l}\text { Anomalous pulmonary venous } \\
\text { connections }\end{array}$ & $6(2.3 \%)$ \\
\hline Truncus arteriosus & $5(1.9 \%)$ \\
\hline Aortic valve disease & $3(1.2 \%)$ \\
\hline Shone's complex & $2(0.8 \%)$ \\
\hline $\begin{array}{l}\text { Congenitally corrected transposi- } \\
\text { tion of the great arteries }\end{array}$ & $2(0.8 \%)$ \\
\hline Atrial septal defect & $2(0.8 \%)$ \\
\hline Pulmonary valve disease & $1(0.4 \%)$ \\
\hline Others/miscellaneous & $(12.5 \%)$ \\
\hline
\end{tabular}

Note: Results depicted in $n$ (percent).

poor nutritional status contributing to the higher rates of EF in this subpopulation. ${ }^{3,5,16,18,21}$

The definition of EF varies among studies, with some defining EF with the need for reintubation within 24 to 96 hours. $^{5,16-18,23}$ This wide range is thought to be due to EF being a slow evolving process in children with CHD as they are capable of compensating for a certain amount of time until requiring the escalation of respiratory support necessitating reintubation. ${ }^{11,14,23}$ Rates of reintubation can be as high as $37 \%$ within the first 48 to 72 hours from initial extubation. ${ }^{5,11,14,23}$ In our study, we defined EF as the need to be reintubated within 48 hours of extubation.

Multiple EF risk factors are unmodifiable such as age, weight, higher STAT scores, CPB and XC time, presence of chromosomal abnormalities, diagnosed genetic syndrome, and duration of MV. Recognizing these risk factors allows the ICU team to anticipate high-risk patients and consider a more conservative approach to their extubation strategy and plan. Reports have shown the association between EF with younger age, and lower weight. 6,11,14,17 Our study supports the association of EF with younger age and lower weight. We show that neonates and infants have a higher rate of EF when compared with older patients. Our study shows that singleventricle physiology, higher STAT scores, longer $\mathrm{CPB}$ and XC times, presence of genetic syndromes, and longer duration of MV were higher in the EF group. Operative mortality was higher in the EF group (8.9 vs. $5.3 \%, p<0.001$ ) (-Table 1 ).

In our univariate analysis, patients who failed extubation when compared to matched controls (2:1) by age, gender and STAT score, risk factors such as CPB, XC time, single-ventricle physiology, duration of MV, and mode of respiratory support post-extubation were not associated with increased odds of EF ( - Table 3). Despite our larger sample size, this supports what prior studies reported as no established link between CPB and XC times and EF has been identified. ${ }^{5,6,11,14,24}$ In a subgroup analysis of neonates, CPB and XC time, and higher STAT score did not achieve statistical significance. CPB time often reflects the complexity of surgery, surgical technique used, patient age and size, and the technical skills of the operator. Although CPB is an essential tool used to conduct cardiac surgery by allowing adequate oxygen delivery to the patients, it is associated with an inflammatory response. This inflammatory response along with reperfusion injury following XC release can result in lung injury that could be exacerbated with MV. All these factors along with patient characteristics and the experience of different members of the ICU staff are important considerations when deciding to extubate a patient following cardiac surgery.

Our study showed that the presence of chromosomal and genetic abnormalities was risk factors for EF. This could be related to the underlying comorbidities, anatomic and dysmorphic features, or neurological impairment related to the syndrome. In particular, heterotaxy syndrome is associated with higher rates of failed extubation in the univariate analysis in the entire cohort (-Table $\mathbf{3}$ ). When performing a literature search, we could not find any prior studies that place patients with heterotaxy syndrome at higher rates of EF following cardiac surgery. What is surprising is patients with DiGeorge and Down Syndrome did not have a higher rate of $\mathrm{EF}$ when compared with other genetic syndromes. Both DiGeorge and Down syndromes are known to have airway abnormalities including but not limited to upper airway malacia, and the unique upper airway anatomy for patients with both syndromes, would put then at higher risk for $\mathrm{EF}^{23}$ One theory is that patients with Down syndrome are more likely to have atrioventricular septal defects and ventricular septal defects (VSD) both requiring surgery at an older age when compared with patients with heterotaxy syndrome who usually carry a cardiac diagnosis of lesions related to single-ventricle physiology, conduction abnormalities associated with other comorbidities. In addition, patients with Down syndrome have a tendency to maintain higher levels of pulmonary vascular resistance following birth for a longer period of time. This tends to be protective to the pulmonary vasculature and delays the development of heart failure symptoms allowing them to grow and require surgery at a later age, usually around 4 to 6 months. These are speculations that will need to be investigated by future studies. If younger age at the time of surgery is thought to be the driving force in patients with heterotaxy syndrome, it is surprising why DiGeorge syndrome is not associated with higher EF rates in our study. Patients with DiGeorge syndrome carry diagnoses such as truncus arteriosus and VSD 
Table 3 Univariate and multivariable analyses for extubation failures $(n=693)$

\begin{tabular}{|c|c|c|c|c|}
\hline \multirow[t]{2}{*}{ Variable } & \multicolumn{2}{|l|}{ Univariate } & \multicolumn{2}{|l|}{ Multivariable } \\
\hline & OR $(95 \% \mathrm{Cl})$ & $p$-Value & OR (95\% Cl) & $p$-Value \\
\hline Weight $(\mathrm{kg})$ & $1.02(0.96-1.09)$ & 0.519 & & \\
\hline \multicolumn{5}{|l|}{ Race } \\
\hline Asian & $0.80(0.31-2.09)$ & 0.650 & & \\
\hline Black/African American & $1.21(0.86-1.69)$ & 0.275 & & \\
\hline Hispanic & $1.07(0.32-3.61)$ & 0.917 & & \\
\hline Other & $1.14(0.47-2.75)$ & 0.774 & & \\
\hline White/Caucasian & Ref. & & & \\
\hline Single ventricle & 1.39 (0.99-1.96) & 0.058 & & \\
\hline CPB time (min) & $1.04(0.99-1.08)$ & 0.106 & & \\
\hline$X C$ time $(\min )$ & $1.01(0.95-1.07)$ & 0.784 & & \\
\hline CICU-LOS (d) & $1.02(1.01-1.03)$ & $<0.001$ & $1.02(1.01-1.03)$ & $<0.001$ \\
\hline Postoperative-LOS (d) & $1.01(1.01-1.02)$ & $<0.001$ & & \\
\hline Hospital-LOS (d) & $1.01(1.01-1.01)$ & $<0.001$ & & \\
\hline Any chromosome abnormality & $0.95(0.64-1.41)$ & 0.788 & & \\
\hline Any genetic syndrome & $1.61(1.13-2.30)$ & 0.009 & $1.50(1.04-2.16)$ & 0.031 \\
\hline \multicolumn{5}{|l|}{ Type of genetic syndrome } \\
\hline DiGeorge syndrome & $1.47(0.73-2.98)$ & 0.280 & & \\
\hline Down syndrome & $0.76(0.37-1.55)$ & 0.450 & & \\
\hline Heterotaxy syndrome & $2.68(1.29-5.58)$ & 0.008 & & \\
\hline Other & $1.39(0.88-2.18)$ & 0.158 & & \\
\hline None & Ref. & & & \\
\hline Duration of MV $(\mathrm{h})^{\mathrm{a}}$ & $1.01(1.00-1.01)$ & 0.202 & & \\
\hline NIPPV & $1.35(0.80-2.30)$ & \multirow[t]{2}{*}{0.264} & & \\
\hline $\mathrm{NC} / \mathrm{HFNC}$ & Ref. & & & \\
\hline
\end{tabular}

Abbreviations: Cl, confidence interval; CICU-LOS, cardiac intensive care unit length of stay; CPB, cardiopulmonary bypass; HFNC, high-flow nasal cannula; Hospital-LOS, hospital length of stay; MV; mechanical ventilation; NC, nasal cannula; NIPPV, non-invasive positive pressure ventilation; OR, odds ratio; postoperative-LOS, postoperative length of stay; STAT score, Society of Thoracic Surgeons and the European Association for CardioThoracic Surgery mortality scores; XC, cross-clamp.

Note: EF cases in entire cohort $(n=231)$. Controls matched by age, gender, STAT score $(n=462)$. Bolded p-Values are statistically significant.

${ }^{a}$ Duration of mechanical ventilation is the time from preoperative intubation to the first attempt of extubation following congenital cardiac surgery.

based on $5 \mathrm{~kg}$ decrease.

'Based on 15-minute increase.

${ }^{\mathrm{d}}$ Based on 12 hours increase.

with interrupted aortic arch type B both of which require surgical repair in the neonatal period.

Prolonged CICU-, post-operative-, and hospital-LOS were all independent risk factors that increased the odds of EF in our study, similar to previous reports. ${ }^{4-6,11,14}$ It is difficult to discern the relationship between EF and CICU-LOS. This issue comes down to "causation and effect" whether the longer duration of MV and CICU-LOS results in EF or if EF results in the longer duration of MV and CICU-LOS. The longer duration of MV prior to initial extubation may reflect chronic lung pathology, deconditioning, or neurological pathology inhibiting extubation and possibly increase the risk of EF. In our center, fast-track extubation protocols are in place and all patients of different ages are managed via these protocols. Deviation from these protocols is the exception and taken case by case depending on the peri-operative and patient factors.

\section{Limitations}

This study is subject to the typical limitations inherent to single-center retrospective cohort studies. As a single-center study, the demographics, clinical characteristics, as well as surgical and medical management of these patients are specific to our institution and might not generalize to other health care systems. Ventilator weaning protocols and extubation readiness testing may be institution specific. The specific inclusion criteria of this study may limit the generalizability of the findings. This study included patients with varying surgical complexity and a wide age range making our 
Pediatric Patients After Cardiac Surgery Beshish et al.

Table 4 Univariate analysis for extubation failures in neonates $(n=318)$

\begin{tabular}{|c|c|c|}
\hline \multirow[t]{2}{*}{ Variable } & \multicolumn{2}{|l|}{ Univariate } \\
\hline & OR $(95 \% \mathrm{Cl})$ & $p$-Value \\
\hline Weight (kg) & $0.68(0.10-4.70)$ & 0.695 \\
\hline \multicolumn{3}{|l|}{ Race } \\
\hline Asian & $1.30(0.30-5.62)$ & 0.725 \\
\hline Black/African American & $1.25(0.75-2.08)$ & 0.384 \\
\hline Hispanic & $0.43(0.05-3.79)$ & 0.450 \\
\hline Other & $1.35(0.43-4.31)$ & 0.608 \\
\hline White/Caucasian & Ref. & \\
\hline Single ventricle & $1.09(0.67-1.76)$ & 0.742 \\
\hline CPB time (min) & $1.02(0.95-1.09)$ & 0.628 \\
\hline XC time (min) & $0.98(0.88-1.08)$ & 0.649 \\
\hline CICU-LOS (d) & $1.02(1.01-1.03)$ & $<0.001$ \\
\hline Postoperative LOS (d) & $1.01(1.01-1.02)$ & $<0.001$ \\
\hline Hospital-LOS (d) & $1.01(1.01-1.02)$ & $<0.001$ \\
\hline $\begin{array}{l}\text { Any chromosome } \\
\text { abnormality }\end{array}$ & $1.07(0.58-1.97)$ & 0.834 \\
\hline Any genetic syndrome & $1.62(0.92-2.84)$ & 0.094 \\
\hline \multicolumn{3}{|l|}{ Type of genetic syndrome } \\
\hline DiGeorge syndrome & $1.30(0.49-3.42)$ & 0.603 \\
\hline Down syndrome & $0.56(0.06-5.05)$ & 0.301 \\
\hline Heterotaxy syndrome & $1.90(0.62-5.86)$ & 0.263 \\
\hline Other & $1.56(0.82-2.99)$ & 0.178 \\
\hline None & Ref. & \\
\hline Duration of MV $(h)^{a}$ & $1.00(1.00-1.01)$ & 0.397 \\
\hline NIPPV & $1.38(0.64-2.97)$ & \multirow[t]{2}{*}{0.417} \\
\hline $\mathrm{NC} / \mathrm{HFNC}$ & Ref. & \\
\hline
\end{tabular}

Abbreviations: $\mathrm{Cl}$, confidence interval; CICU-LOS, cardiac intensive care unit length of stay; CPB, cardiopulmonary bypass; HFNC, high-flow nasal cannula; Hospital-LOS, hospital length of stay; MV; mechanical ventilation; NC, nasal cannula; NIPPV, non-invasive positive pressure ventilation; OR, odds ratio; postoperative-LOS, postoperative length of stay; STAT score, Society of Thoracic Surgeons and the European Association for Cardio-Thoracic Surgery mortality score; XC, cross-clamp.

Note: EF cases in neonates $(n=106)$. Controls matched by age, gender, and STAT score $(n=212)$. Bolded p-Values are statistically significant. ${ }^{a}$ Duration of mechanical ventilation is the time from preoperative intubation to the first attempt of extubation following congenital cardiac surgery.

(Multivariable logistic regression of the neonate-only cohort was attempted but did not achieve statistical significance).

${ }^{b}$ Based on $5 \mathrm{~kg}$ decrease.

'Based on 15-minute increase.

${ }^{\mathrm{d}}$ Based on 12 hours increase.

cohort nonhomogeneous; however, these larger numbers allow for the increased power of the findings. Despite these limitations, the prevalence of $\mathrm{EF}$ in the entire cohort, in general, and in the neonatal population, in particular, are lower but still comparable to studies that are published in the field. However, our outcomes and possible risk factors need to be confirmed by larger multicenter prospective studies.

\section{Conclusion}

This study demonstrates that EF after congenital cardiac surgery is associated with younger age, lower weight, single-ventricle physiology, longer $\mathrm{CPB}$ and $\mathrm{XC}$ times, and genetic syndromes. $\mathrm{EF}$ is a slow evolving process in children with CHD as they are capable of compensating for a certain amount of time until requiring escalation of respiratory support necessitating reintubation. Patients failing extubation have longer lengths of CICU and hospital stay and higher mortality. Recognition of these risk factors may provide clinicians the ability to identify patients at high risk for EF allowing for more rapid reintervention to limit adverse outcomes.

\section{Conflict of Interest}

None declared.

\section{References}

1 Kloth RL, Baum VC. Very early extubation in children after cardiac surgery. Crit Care Med 2002;30(04):787-791

2 Mittnacht AJ, Thanjan M, Srivastava S, et al. Extubation in the operating room after congenital heart surgery in children. J Thorac Cardiovasc Surg 2008;136(01):88-93

3 Davis S, Worley S, Mee RB, Harrison AM. Factors associated with early extubation after cardiac surgery in young children. Pediatr Crit Care Med 2004;5(01):63-68

4 Harris KC, Holowachuk S, Pitfield S, et al. Should early extubation be the goal for children after congenital cardiac surgery? J Thorac Cardiovasc Surg 2014;148(06):2642-2647

5 Miura S, Hamamoto N, Osaki M, Nakano S, Miyakoshi C. Extubation failure in neonates after cardiac surgery: prevalence, etiology, and risk factors. Ann Thorac Surg 2017;103(04):1293-1298

6 Manrique AM, Feingold B, Di Filippo S, Orr R, Kuch BA, Munoz R. Extubation after cardiothoracic surgery in neonates, children, and young adults: one year of institutional experience. Pediatr Crit Care Med 2007;8(06):552-555

7 Preisman S, Lembersky H, Yusim Y, et al. A randomized trial of outcomes of anesthetic management directed to very early extubation after cardiac surgery in children. J Cardiothorac Vasc Anesth 2009;23(03):348-357

8 Yamasaki Y, Shime N, Miyazaki T, Yamagishi M, Hashimoto S, Tanaka Y. Fast-track postoperative care for neonatal cardiac surgery: a single-institute experience. J Anesth 2011;25(03): 321-329

9 Kurihara Y, Shime N, Miyazaki T, Hashimoto S, Tanaka Y. Clinical and hemodynamic factors associated with the outcome of early extubation attempts after right heart bypass surgery. Interact Cardiovasc Thorac Surg 2009;8(06):624-628

10 Garg R, Rao S, John C, et al. Extubation in the operating room after cardiac surgery in children: a prospective observational study with multidisciplinary coordinated approach. J Cardiothorac Vasc Anesth 2014;28(03):479-487

11 Gupta P, McDonald R, Gossett JM, et al. A single-center experience of extubation failure in infants undergoing the Norwood operation. Ann Thorac Surg 2012;94(04):1262-1268

12 Harrison AM, Cox AC, Davis S, Piedmonte M, Drummond-Webb JJ Mee RB. Failed extubation after cardiac surgery in young children: prevalence, pathogenesis, and risk factors. Pediatr Crit Care Med 2002;3(02):148-152 
13 Kanter RK, Bove EL, Tobin JR, Zimmerman JJ. Prolonged mechanical ventilation of infants after open heart surgery. Crit Care Med 1986;14(03):211

14 Gupta P, McDonald R, Goyal S, et al. Extubation failure in infants with shunt-dependent pulmonary blood flow and univentricular physiology. Cardiol Young 2014;24(01):64-72

15 Thiagarajan RR, Bratton SL, Martin LD, Brogan TV, Taylor D. Predictors of successful extubation in children. Am J Respir Crit Care Med 1999;160(5 Pt 1):1562-1566

16 Kurachek SC, Newth CJ, Quasney MW, et al. Extubation failure in pediatric intensive care: a multiple-center study of risk factors and outcomes. Crit Care Med 2003;31(11):2657-2664

17 Baisch SD, Wheeler WB, Kurachek SC, Cornfield DN. Extubation failure in pediatric intensive care incidence and outcomes. Pediatr Crit Care Med 2005;6(03):312-318

18 Fontela PS, Piva JP, Garcia PC, Bered PL, Zilles K. Risk factors for extubation failure in mechanically ventilated pediatric patients. Pediatr Crit Care Med 2005;6(02):166-170
19 Epstein SK, Ciubotaru RL, Wong JB. Effect of failed extubation on the outcome of mechanical ventilation. Chest $1997 ; 112(01)$ : 186-192

20 Torres A, Gatell JM, Aznar E, et al. Re-intubation increases the risk of nosocomial pneumonia in patients needing mechanical ventilation. Am J Respir Crit Care Med 1995;152(01):137-141

21 Edmunds S, Weiss I, Harrison R. Extubation failure in a large pediatric ICU population. Chest 2001;119(03):897-900

22 O'Brien SM, Clarke DR, Jacobs JP, et al. An empirically based tool for analyzing mortality associated with congenital heart surgery. J Thorac Cardiovasc Surg 2009;138(05):1139-1153

23 Laudato N, Gupta P, Walters HL III, Delius RE, Mastropietro CW. Risk factors for extubation failure following neonatal cardiac surgery. Pediatr Crit Care Med 2015;16(09):859-867

24 Bandla HP, Hopkins RL, Beckerman RC, Gozal D. Pulmonary risk factors compromising postoperative recovery after surgical repair for congenital heart disease. Chest 1999;116(03): $740-747$ 